\title{
Fulvestrant reverses doxorubicin resistance in multidrug-resistant breast cell lines independent of estrogen receptor expression
}

\author{
YUAN HUANG $^{1,2^{*}}$, DONGHAI JIANG ${ }^{2 *}$, MEIHUA SUI ${ }^{3}$, XIAOJIA WANG $^{1}$ and WEIMIN FAN ${ }^{2,4}$ \\ ${ }^{1}$ Department of Chemotherapy, Zhejiang Cancer Hospital, Hangzhou, Zhejiang 310022; \\ ${ }^{2}$ Program of Innovative Cancer Therapeutics, Key Laboratory of Combined Multi-Organ Transplantation, \\ Ministry of Public Health, First Affiliated Hospital of Zhejiang University School of Medicine, Hangzhou, \\ Zhejiang 310006; ${ }^{3}$ Center for Cancer Biology and Innovative Therapeutics, Clinical Research Institute, \\ Zhejiang Provincial People's Hospital, Hangzhou, Zhejiang 310014, P.R. China; ${ }^{4}$ Department of Pathology \\ and Laboratory Medicine, Medical University of South Carolina, Charleston, SC 29425-9080, USA
}

Received June 5, 2016; Accepted October 31, 2016

DOI: $10.3892 /$ or.2016.5315

\begin{abstract}
Drug resistance, a major obstacle to successful cancer chemotherapy, frequently occurs in recurrent or metastatic breast cancer and results in poor clinical response. Fulvestrant is a new type of selective estrogen receptor (ER) downregulator and a promising endocrine therapy for breast cancer. In this study, we evaluated the combination treatment of fulvestrant and doxorubicin in ER-negative multidrugresistant (MDR) breast cancer cell lines Bads-200 and Bats-72. Fulvestrant potentiated doxorubicin-induced cytotoxicity, apoptosis and G2/M arrest with upregulation of cyclin B1. It functioned as a substrate for P-glycoprotein (P-gp) without affecting its expression. Furthermore, fulvestrant not only restored the intracellular accumulation of doxorubicin but also relocalized it to the nuclei in Bats- 72 and Bads-200 cells, which may be another potential mechanism of reversal of P-gp mediated doxorubicin resistance. These results indicated that the combination of fulvestrant and doxorubicin-based chemotherapy may be feasible and effective for patients with advanced breast cancer.
\end{abstract}

\section{Introduction}

Breast cancer is one of the most common malignancies and a leading cause of cancer-related mortality in women (1).

Correspondence to: Professor Weimin Fan, Program of Innovative Cancer Therapeutics, Key Laboratory of Combined Multi-Organ Transplantation, Ministry of Public Health, First Affiliated Hospital of Zhejiang University School of Medicine, 79 Qingchun Road, Hangzhou, Zhejiang 310006, P.R. China

E-mail: fanw@zju.edu.cn

*Contributed equally

Key words: breast cancer, estrogen receptor, fulvestrant, doxorubicin, drug resistance
Hormone receptor (HR), especially estrogen receptor (ER), plays important roles in the development and progression of breast cancer (2). There are different therapy choices in clinic according to HR status of breast cancer. The HR-positive subtype which requires estrogen to grow potentially is susceptible to endocrine therapy that blocks the receptors to improve the prognosis $(3,4)$, while the HR-negative sub-type, mostly relys on traditional chemotherapy. For example, anthracycline and taxanes based regimens are widely used as the first-line scheme $(5,6)$. Although HR-negative breast cancer is sensitive to chemotherapy in initial treatment (7), tumor recurrence frequently occurs (8). In fact, drug resistance is believed to be one of the most common causes of tumor recurrence and is associated with a poor outcome for HR-negative breast cancer patients.

Women with recurrent or metastatic HR-positive breast cancer are appropriate candidates for initial endocrine therapy, and endocrine therapy may be active in patients with negative HR examination, especially in soft tissue disease and/ or bone-dominant disease (9-11). Endocrine therapy is also associated with relatively low toxicity. However, to date, endocrine therapy and chemotherapy are recommended to be given sequentially, there is little evidence supporting the combination of endocrine therapy and chemotherapy as the ideal therapy strategy. Fulvestrant (ICI 182,780, Faslodex) is a new type of selective ER downregulator (12-14). It binds, blocks and degrades ER, then inhibits ER-mediated transcriptional activity. Considering that fulvestrant is indicated for patients with disease progression which may imply the development of aquired drug resistance, we wonder whether fulvestrant could further enhance efficacy in combination regimens. Several research groups have reported the rationale and evidence for the efficacy of fulvestrant in combination with other agents such as gefitinib and trastuzumab $(15,16)$.

In our previous studies, we found that the combination of fulvestrant could markedly reverse the ER-mediated resistance and sensitize ER-positive BCap37 cells which were derived from stable transfection of an ER- $\alpha$ expression vector into ER-negative BCap37 cells to antimicrotubule agents such as paclitaxel and vinca alkaloids in vitro and in vivo (17-19). So 
fulvestrant not only works as endocrine therapy but can also sensitize the efficacy of conventional chemotherapeutic drugs for ER-positive breast cancer. More recently, we successfully established two independent novel paclitaxel-resistant cell lines Bats-72 and Bads-200 from the same parental BCap37 cell line, both of which were ER-negative and showed crossresistance to other anticancer drugs including doxorubicin (20). Compared to parental BCap37 cells, both Bads-200 and Bats-72 cells overexpress P-glycoprotein (P-gp), which functions as an ATP-dependent efflux pump with a variety of substrates and plays an important role in mediating multidrug resistance (21-23). Interestingly, we found that fulvestrant could significantly reverse the resistance to paclitaxel in Bads-200 and Bats-72 cell lines. In addition, we also found fulvestrant could enhance their sensitivity to many other chemotherapy drugs including docetaxel, vinorelbine and doxorubicin (24).

To further explore this interesting phenomenon, we performed a series of experiments to investigate the combination treatment of fulvestrant and doxorubcin in ER-negative breast cancer cell lines Bads-200 and Bats-72 which may support the feasibility of the combination of fulvestrant and chemotherapeutic drugs for MDR breast cancer in the clinic.

\section{Materials and methods}

Cell lines and cell culture. The human breast cancer cell line BCap37, two MDR cell lines Bads-200 and Bats-72, paclitaxelselected derivative obtained from parental BCap37 cell line (20), the human oral squamous carcinoma cell line KB and and its vincristine-selected derivative KBv200 were cultured in RPMI-1640 medium with $10 \%$ fetal bovine serum and $1 \%$ penicillin/streptomycin. Bads-200 cells were maintained in medium containing $200 \mathrm{nM}$ paclitaxel (20), and KBv200 were grown in medium added $100 \mathrm{nM}$ vincristine to keep their drug resistance characteristics (25).

Drugs and treatments. Doxorubicin, fulvestrant, verapamil and tamoxifen were purchased from Sigma (St. Louis, MO) for studies in vitro. All chemicals were prepared according to the drug specifications and diluted with culture medium to the desired concentrations before use. All cells were cultured in drug-free medium for more than $24 \mathrm{~h}$ before treatments. Then the cells were treated with distinct dose of doxorubicin with or without 3-h pretreatment of fulvestrant.

In vitro cytotoxicity assays. The 3-(4,5-dimethylthiazol-2-yl)2,5-diphenyltetrazolium bromide (MTT) assay was used to measure the drug-induced cytotoxicity. Briefly, $10^{4}$ cells/well were seeded and incubated overnight, varying concentrations of designated drugs were added into each well. At the end of drug exposure for $72 \mathrm{~h}$, MTT solution was added and the plates were further incubated for $3 \mathrm{~h}$. Then, the medium was removed and $200 \mu \mathrm{l}$ of DMSO was added to dissolve the formazan crystals, then individual wells were determined at $570 \mathrm{~nm}$ with a microplate reader. The relative fraction of survival was calculated by dividing the absorbance of treated wells by that of the untreated control. Background absorbencies were subtracted, $\mathrm{IC}_{50}$ values represent concentrations causing $50 \%$ inhibition of cell growth.
Cell cycle analysis. Cell cycle distributions were assessed by flow cytometric analysis. Cells were incubated in 6-well plates with $10^{5}$ cells/well. After $48 \mathrm{~h}$ of drug treatment, Both floating and adherent cells were collected and washed twice with ice-cold PBS. Following fixation in $70 \%$ ethanol diluted in PBS, the fixed cells were washed twice with PBS and treated with $100 \mu \mathrm{g} / \mathrm{ml}$ RNase and $40 \mu \mathrm{g} / \mathrm{ml}$ propidium iodide at room temperature for 0.5-1.0 $\mathrm{h}$ in the dark. Cell cycle distribution and DNA content were tested by a Coulter Epics V instrument (Beckman Coulter, Inc., Fullerton, CA, USA) with an argon laser set to excite at $488 \mathrm{~nm}$.

Intracellular doxorubicin distribution and accumulation. Confocal cell images were determined to assess intracelluar doxorubicin distribution and accumulation. Cells were seeded on coverslips in 6-well tissue culture plates and incubated for $48 \mathrm{~h}$ to grow as monolayers, then they were treated with or without $5 \mu \mathrm{M}$ fulvestrant for $2 \mathrm{~h}$ before exposure with $5 \mu \mathrm{M}$ doxorubicin. After washing twice with ice-cold PBS, $10 \mu \mathrm{g} / \mathrm{ml}$ Hoechst-33342 was added into plates for nuclear staining, followed by fixation with $4 \%$ paraformaldehyde solution. Finally, air-dried coverslips were mounted on slides with glycerol-PBS (1:1) and imaged using a confocal laser scanning microscope at 600 times magnification.

Quantitation of doxobicin uptake and efflux. Cells were plated into 6-well plates and allowed to grow for $48 \mathrm{~h}$. Then cells were exposed with doxorubicin in the presence or absence of fulvestrant followed by incubation for $2 \mathrm{~h}$. To evaluate doxorubicin efflux, cells treated $2 \mathrm{~h}$ with the combination of doxorubicin and fulvestrant were further incubated in drug-free medium with or without fulvestrant for additional $2 \mathrm{~h}$. After washed with ice-cold PBS, intracellular doxorubicin fluorescent intensity (accumulation fluorescence) was determined with Coulter Epics V instrument (Beckman Coulter, Inc.). The cells were excited at $485 \mathrm{~nm}$, and emission was collected at $530 \mathrm{~nm}$ for doxorubicin.

ATPase activity assay. P-gp ATPase activity was measured with the Pgp-Glo assay system according to the manufacturer's instructions (Promega, Madison, WI, USA) to identify the impact of fulvestrant on P-gp ATPase activity (26). Fulvestrant $(5 \mu \mathrm{M})$ or $5 \mu \mathrm{M}$ doxorubicin were incubated with $5 \mathrm{mM}$ MgATP and $25 \mu \mathrm{g}$ recombinant human P-gp membranes at $37^{\circ} \mathrm{C}$ for $40 \mathrm{~min}$. Luminescence was initiated by ATP detection buffer. After 20 min to develop luminescent signals, the multiplate was read on a plate-reading luminometer. The decreased luminescence reflects ATP consumption.

Apoptosis assay. Cells were treated with doxorubicin and fulvestrant alone or in combination for $48 \mathrm{~h}$ and the cell morphology was identified using a light microscope at $\mathrm{x} 400$ magnification. Annexin V-FITC staining (Beyotime, Haimen, China) was used to detect cell apoptosis according to the manufacturer's instructions (27). Briefly, after the designated treatment and at the end of time-point, both detached and attached cells were harvested and washed twice with ice-cold PBS. The collected cells were then resuspended with Annexin V binding buffer and incubated with $5 \mu \mathrm{l}$ of fluorescein isothiocyanate (FITC) Annexin $\mathrm{V}$ for $15 \mathrm{~min}$ at $4^{\circ} \mathrm{C}$ in the dark. The percentages of apoptotic cells were determined by flow cytometry. 


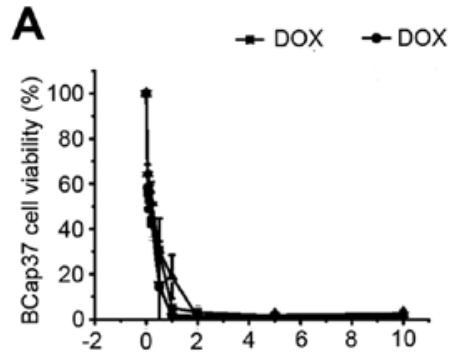

Concentrations of Doxorubicin $(\mu \mathrm{M})$

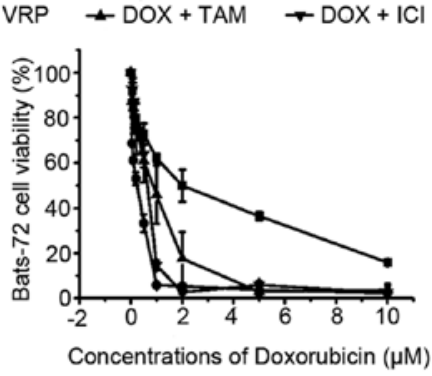

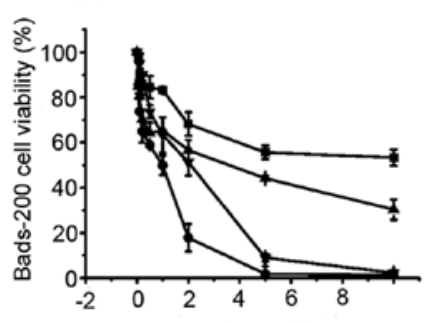

Concentrations of Doxorubicin $(\mu \mathrm{M})$
B

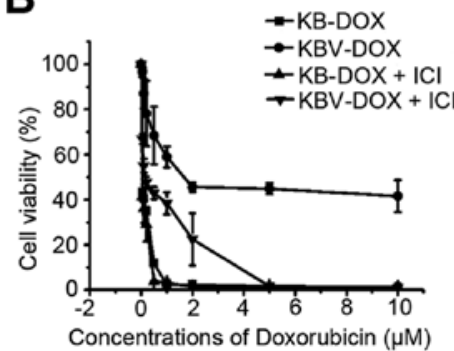

C

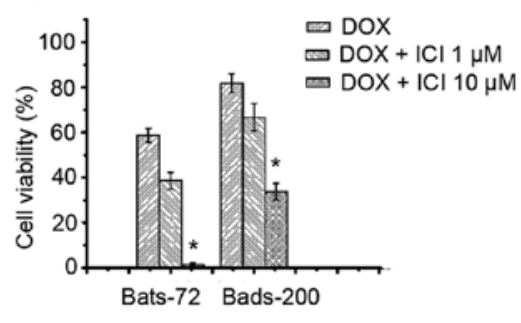

Figure 1. Fulvestrant sensitizes doxorubicin-induced cytotoxicity in ER-negative MDR-positive cell lines. Cells were exposed to designated concentrations of doxorubicin with or without co-treatment of fulvestrant or P-gp modulators for $72 \mathrm{~h}$, the cell viabilities were determined by MTT assays. (A) The reversal activity of $5 \mu \mathrm{M}$ fulvestrant among BCap37, Bats-72 and Bads-200 cells compared with other P-gp modulators (5 $\mu \mathrm{M}$ verapamil and $5 \mu \mathrm{M}$ tamoxifen). (B) The reversal effect of $5 \mu \mathrm{M}$ fulvestrant on $\mathrm{KB} / \mathrm{KBv} 200$ cells (known P-gp mediated resistance). (C) Potency of different fulvestrant concentrations (1 and $10 \mu \mathrm{M}$ ) in enhancing doxorubicin cytotoxicity in Bats-72, Bads-200 cells. * P<0.05 vs. DOX + ICI $1 \mu$ M. DOX, doxorubicin; ICI, fulvestrant; VRP, verapamil; TAM, tamoxifen; KBV, KBv200.

Western blotting. Cells were treated with doxorubicin and fulvestrant alone or in combination for $24 \mathrm{~h}$. Cellular protein was isolated with a protein extraction buffer (Beyotime). Protein concentrations were measured with the BCA protein assay kit (Pierce). Equal amounts (40 $\mu \mathrm{g} /$ lane) of proteins were fractionated on 10-12\% SDS-PAGE gels and transferred to polyvinylidene difluoride membranes. The membranes were incubated with the desired primary antibodies, respectively. After washing with PBS containing $0.1 \%$ (v/v) Tween-20, the membranes were incubated with anti-mouse or anti-rabbit IgG coupled to HRP second antibodies for $2 \mathrm{~h}$ at room temperature followed by enhanced chemiluminescent staining using the ECL system. $\beta$-actin was used for normalization of protein loading.

Statistical analysis. Data are presented as mean \pm standard error (SE). Student's t-test was used to determine the statistical difference for two-group comparisons, and multiple-treatment groups were analyzed by one-way ANOVA. Differences were considered statistically significant at a level of $\mathrm{P}<0.05$.

\section{Results}

Fulvestrant sensitizes doxorubicin-induced cytotoxicity in ER-negative MDR cell lines. To evaluate the modulation activity of fulvestrant to doxorubicin in ER-negative MDR cell lines, including Bats-72, Bads-200 cell lines and KBv200 cell lines all with MDR phenotype as a result of P-gp overexpression, we examined its intrinsic cytotoxicity. The survival rates of the two MDR cell lines were $>90 \%$ after exposure to $1-10 \mu \mathrm{M}$ fulvestrant for $72 \mathrm{~h}$ (data not shown) and we chose $5 \mu \mathrm{M}$ fulvestrant for the following test to evaluate its reversal activity. The sensitivities of the two different MDR cell lines treated with a series of concentrations of doxorubicin in the absence or presence of $5 \mu \mathrm{M}$ fulvestrant are shown in Fig. $1 \mathrm{~A}$ and $\mathrm{B}$. The $\mathrm{IC}_{50}$ values of 72-h doxorubicin exposure approximately were $0.11 \pm 0.03,1.91 \pm 0.17$ and $10.97 \pm 3.86 \mu \mathrm{M}$ respectively. Three-hour-pretreatment with fulvestrant, which alone had no effect on cell viability, significantly sensitized Bats-72 and Bads-200 to doxorubicin in a dose-dependent manner. The $\mathrm{IC}_{50}$ values were decreased to $0.50 \pm 0.10 \mu \mathrm{M}$ in Bats72 and 1.47 $\pm 0.05 \mu \mathrm{M}$ in Bads200, respectively. For KB, $\mathrm{KBv} 200$ cells, similar results were found, the $\mathrm{IC}_{50}$ value for $\mathrm{KBv} 200$ cells was decreased from $2.30 \pm 0.9$ to $0.20 \pm 0.03 \mu \mathrm{M}$ after treated with doxorubicin alone or in combination with fulvestrant for $72 \mathrm{~h}$ (Fig. 1B and Table I). Other doses of fulvestrant were selected to further determine whether the reversal potency is dose-dependent. As Fig. 1C shows, $10 \mu \mathrm{M}$ fulvestrant produced a more significant reversal effect than $1 \mu \mathrm{M}$ fulvestrant after Bats72 and Bads200 cells were cotreated with $1 \mu \mathrm{M}$ doxorubicin, although $1 \mu \mathrm{M}$ fulvestrant also can enhance doxorubicin-induced cytotoxicity. These data indicated that fulvestrant strongly sensitized doxorubicininduced cytotoxicity in MDR cell lines.

The reversal activity of Fulvestrant was further compared with the classic P-gp modulators' efficacies, like verapamil and tamoxifen. First the cytotoxicities of verapamil and tamoxifen alone were determined by MTT assay, their concentrations at $\leq 10 \mu \mathrm{M}$ exerted slight cytotoxicity on BCap37, Bats-72, Bads-200 cells, and the cell survival rates were $>90 \%$ (data not shown). Verapamil $(5 \mu \mathrm{M})$ or tamoxifen were co-treated with serial concentrations of doxorubicin to BCap37, Bats-72, Bads-200 cells, respectively. As shown in Fig. 1A and Table I, verapamil reduced the $\mathrm{IC}_{50}$ values of doxorubicin approximately 
Table I. The reversal activities of fulvestrant and other P-gp modulators.

\begin{tabular}{lccccc}
\hline & \multicolumn{5}{c}{$\mathrm{IC}_{50}(\mu \mathrm{m})^{\mathrm{b}}$} \\
\cline { 2 - 6 } Drug $^{\mathrm{a}}$ & BCap37 & Bats-72 & Bads-200 & KB & KBv200 \\
\hline DOX & $0.11 \pm 0.03$ & $1.91 \pm 0.17$ & $10.97 \pm 3.86$ & $0.05 \pm 0.01$ & $2.30 \pm 0.91$ \\
DOX+ICI & $0.13 \pm 0.04$ & $0.50 \pm 0.10$ & $1.47 \pm 0.05$ & $0.05 \pm 0.01$ & $0.20 \pm 0.03$ \\
DOX+VRP & $0.09 \pm 0.03$ & $0.17 \pm 0.08$ & $1.20 \pm 0.01$ & - & - \\
DOX+TAM & $0.15 \pm 0.02$ & $0.86 \pm 0.13$ & $4.26 \pm 0.70$ & - & - \\
\hline
\end{tabular}

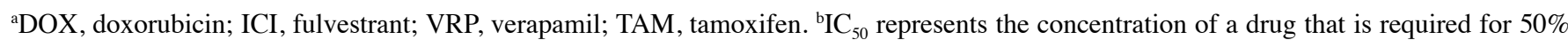
inhibition of cell growth.

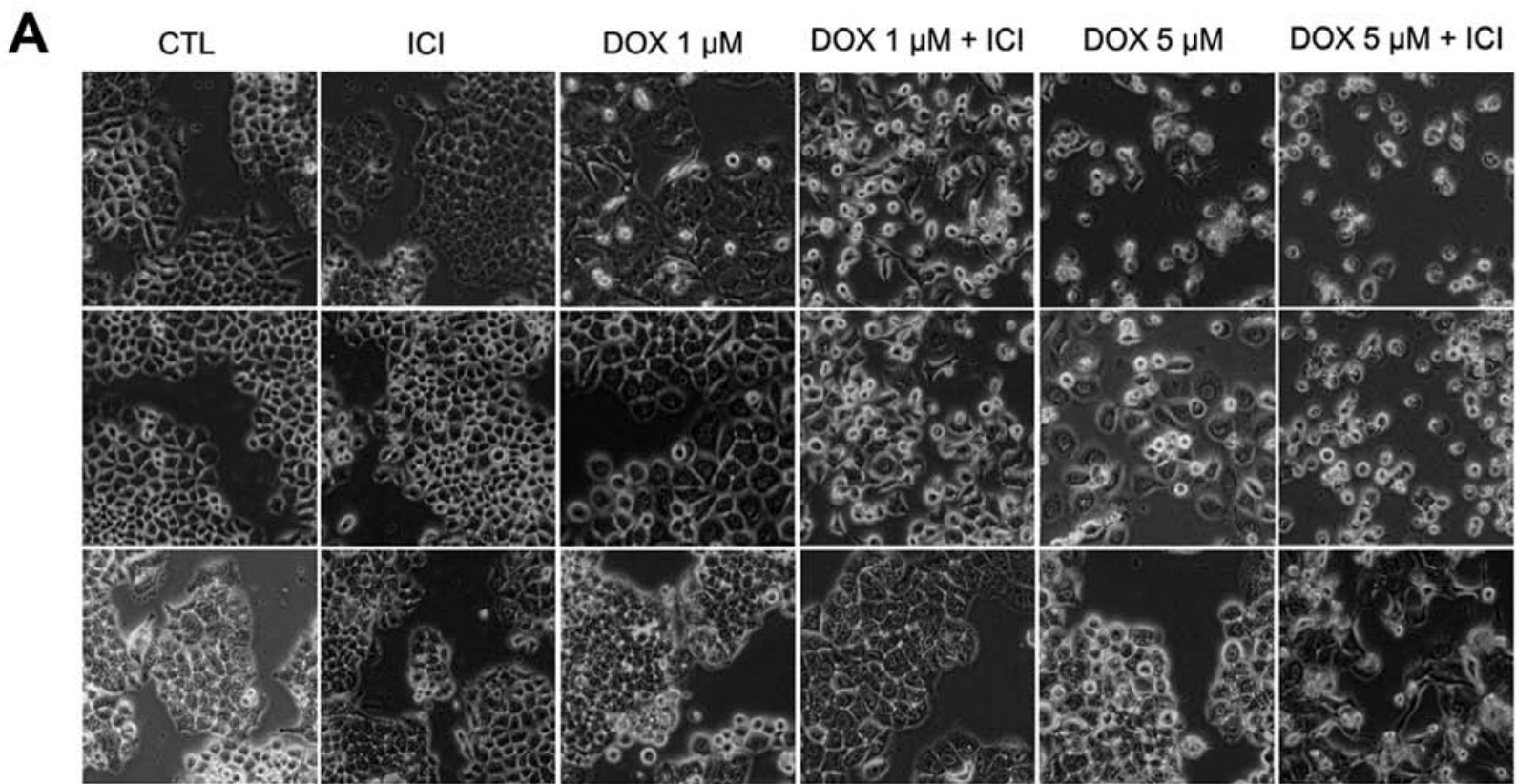

BCap37

Bats-72

Bads-200

B

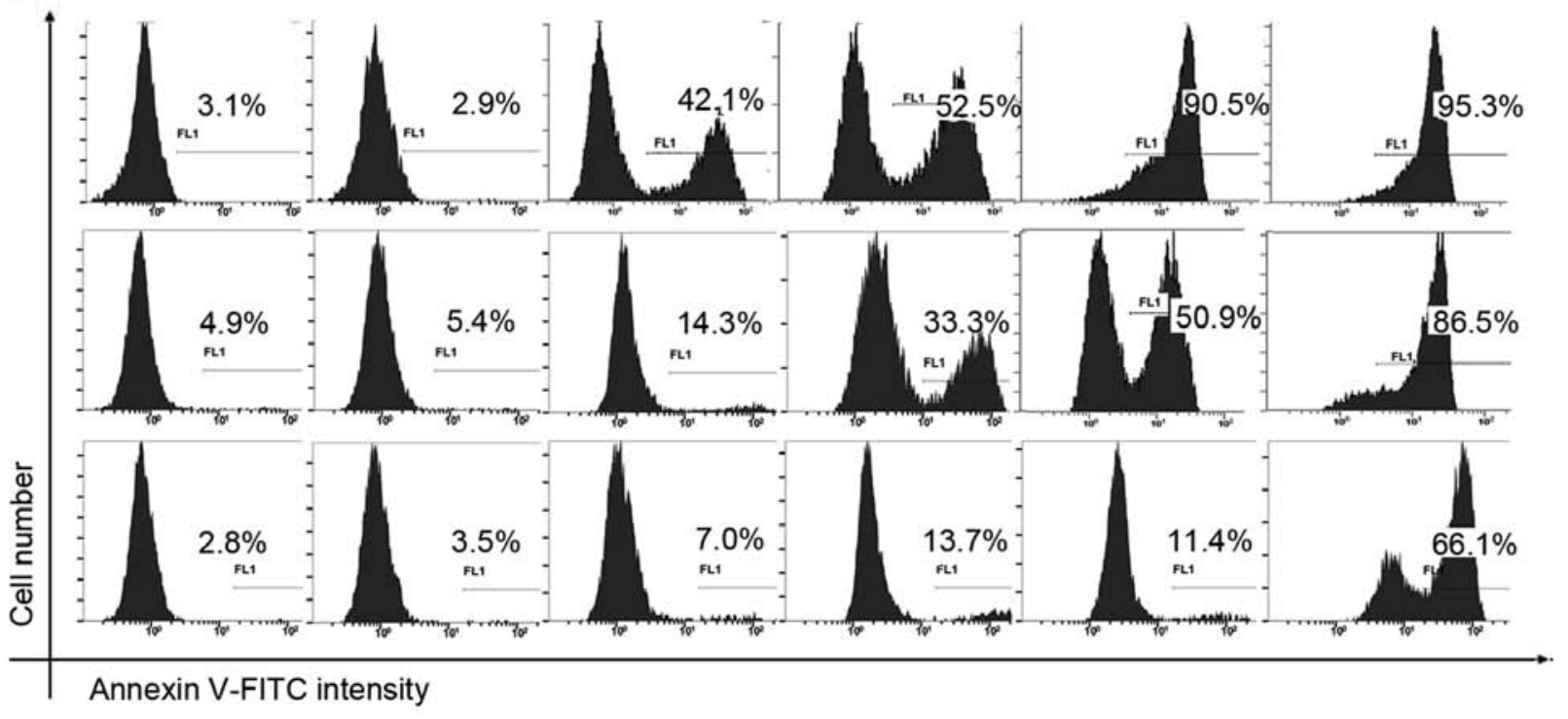

BCap37

Bats-72

Bads-200

Figure 2. Fulvestrant potentiates doxorubicin-induced apoptosis. BCap37, Bats-72 and Bads-200 cells treated with fulvestrant $(5 \mu \mathrm{M})$, doxorubicin (1 or $5 \mu \mathrm{M}$ ) or their combination for $48 \mathrm{~h}$. (A) Apoptotic cells by morphologic analysis. Cells were photographed at 400 -fold magnification using a regular microscope. (B) Doxorubicin-induced apoptosis was quantified by Annexin V-FITC staining. BCap37, Bats-72 and Bads-200 cells were harvested and stained with Annexin V-FITC for flow cytometric analysis. The fractions of Annexin V-FITC positive cells indicate the ratios of apoptotic cells. CTL, control; ICI, fulvestrant; DOX, doxorubicin. 
A

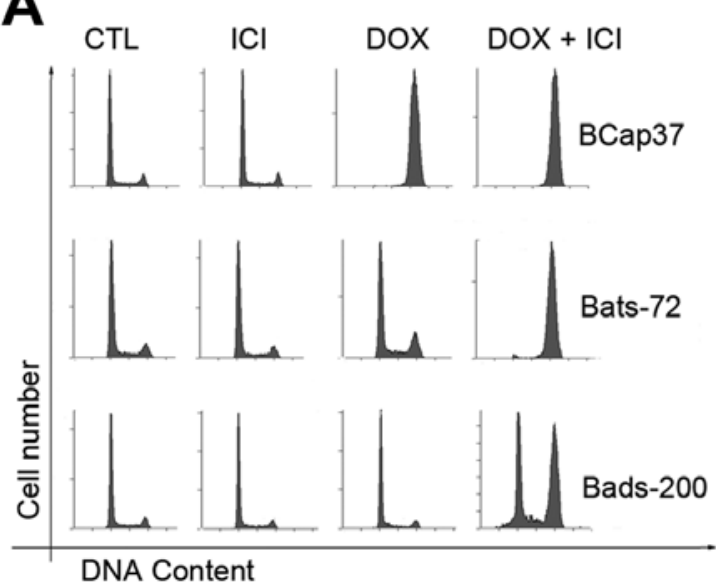

B

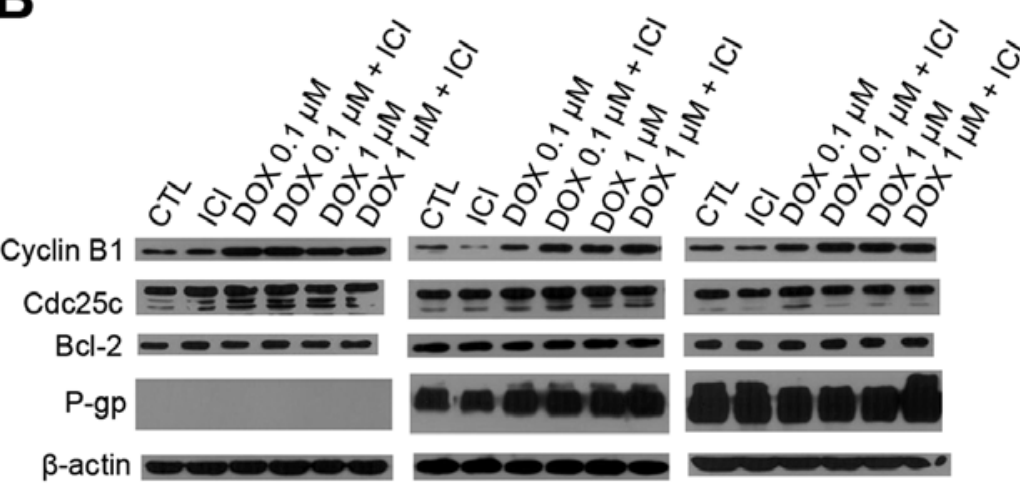

BCap37
Bats-72
Bads-200

Figure 3. Fulvestrant enhances doxorubicin-induced G2/M arrest and upregulation of cyclin B1. (A) Flow cytometric analysis of cell cycle distribution. BCap37, Bats-72 and Bads-200 cells treated with fulvestrant $(5 \mu \mathrm{M})$, doxorubicin $(0.1 \mu \mathrm{M})$ or their combination for $24 \mathrm{~h}$ were harvested and stained with propidium iodide DNA content analysis. The peaks corresponding to G0/G1 and G2/M phases of cell cycle are shown. (B) Western blot analyses for the cyclin B1, Cdc25c, Bcl-2 and P-gp. Whole-cell proteins were extracted from cells treated with fulvestrant $(5 \mu \mathrm{M})$, doxorubicin $(0.1$ or $1 \mu \mathrm{M})$ or their combination for $24 \mathrm{~h}$. Equal amounts $(40 \mu \mathrm{g} / \mathrm{lane})$ of cellular protein were analyzed. $\beta$-actin protein was blotted as a control. CTL, control; ICI, fulvestrant; DOX, doxorubicin .

to $0.17 \pm 0.08$ and $1.20 \pm 0.01 \mu \mathrm{M}$ in Bats72 and Bads200 cells, respectively, and the $\mathrm{IC}_{50}$ values of doxorubicin in the presence of tamoxifen were $0.86 \pm 0.13$ and $4.26 \pm 0.70 \mu \mathrm{M}$ in Bats72 and Bads 200 cells, respectively. The trend of cell survival curves also showed that the reversal potency of fulvestrant is similar to that of verapamil and is more effective than that of tamoxifen when in combination with doxorubicin at the same doses.

Fulvestrant potentiates doxorubicin-induced apoptosis. To further investigate whether fulvestrant potentiates the cytotoxicity of doxorubicin to induce apoptosis, morphologic analysis was done using a regular light microscope after BCap37, Bats-72, Bads-200 cells treated with doxorubicin and fulvestrant alone or in combination for $48 \mathrm{~h}$. As depicted in Fig. 2, fulvestrant reinforced the degree of doxorubicininduced cell death in Bats-72 and Bads-200 cells, while treatment with fulvestrant alone showed no change on cellular morphology. Quantification of apoptosis was determined by Annexin V-FITC assay. $5 \mu \mathrm{M}$ fulvestrant significantly increased the percentage of Annexin V-positive cells after treated with $1 \mu \mathrm{M}$ or $5 \mu \mathrm{M}$ doxorubicin in Bats 72 cells and Bads200 cells. Interestingly, fulvestrant also increased apoptosis induced by $1 \mu \mathrm{M}$ doxorubicin in Bcap37 cells and obviously changed their cellular morphology, while there was little difference between treatment with $5 \mu \mathrm{M}$ doxorubicin in the presence or absence of fulvestrant, both of which induced $>90 \%$ percentage of Bcap37 cells death, as a result that $5 \mu \mathrm{M}$ doxorubicin alone killed almost all the Bcap37 cells. The results demonstrate that the apoptosis levels increased with higher doxorubicin concentrations and fulvestrant potentiated doxorubicin-induced apoptotic in the three cell lines.

Fulvestrant enhances doxorubicin-induced G2/M arrest and upregulation of cyclin B1. In previous studies, different levels of doxorubicin dose produced different cell death pathways, and lower doses may induce $\mathrm{G} 2 / \mathrm{M}$ arrest $(28,29)$. To investigate whether fulvestrant also enhanced doxorubicin-induced G2/M arrest, cell cycle distribution was analysed by flow cytometric assay (Fig. 3A). After 48-h exposure of $0.1 \mu \mathrm{M}$ doxorubicin with 3-h pretreatment of $5 \mu \mathrm{M}$ fulvestrant, the population of cells in G2/M phase in the Bats72 and Bads200 increased markedly compared with that of the doxorubicin treatment alone. Conversely, fulvestrant showed little effect on the cell cycle of BCap37 cells while the majority of BCap37 cells were arrested in $\mathrm{G} 2 / \mathrm{M}$ phases with or without fulvestrant.

In order to further elucidate that fulvestrant increased doxorubicin-induced cell cycle arrest at G2/M phase, we examined its modulation on protein levels associated with the G2/M phase of the cell cycle. BCap37, Bats-72 and Bads-200 cells were exposed to 0.1 and $1 \mu \mathrm{M}$ doxorubicin for $24 \mathrm{~h}$, and then the levels of several protein were detected. Western blotting (Fig. 3B) revealed fulvestrant upregulated cyclin B1 expression following co-treatment with $0.1 \mu \mathrm{M}$ doxorubicin in Bats-72 cells and both 0.1 and $1 \mu \mathrm{M}$ doxorubicin in Bads-200 cells, while $0.1 \mu \mathrm{M}$ doxorubicin resulted in the maximal expression levels of cyclin B1 in BCap37 cells with or without fulvestrant. Cyclin B1 expression seems cell cycle-dependent, the increase in cyclin B1 protein levels was associated with the extent of doxorubicin-induced G2/M arrest in all the three cell lines. Another protein, the $\mathrm{cdc} 25 \mathrm{c}$ which is an upstream regulator of cyclin B1 showed no change in protein levels compared with various treatment, further indicating that the doxorubicin-induced G2/M arrest was more likely mediated by the level of cyclin B1. Additionally, we also detected the anti-apoptotic Bcl-2 protein levels which remained constant before and after treatment.

Fulvestrant functions as a substrate for transport by P-gp, without affecting its expression. It has been believed that the change of P-gp expression or its function may influence the efficacy of P-gp-mediated MDR, therefore the relationship between fulvestrant and P-gp was studied. Western blotting (Fig. 3B) showed doxorubicin, fulvestrant alone or in combination did not alter the expression of P-gp. To further determine 


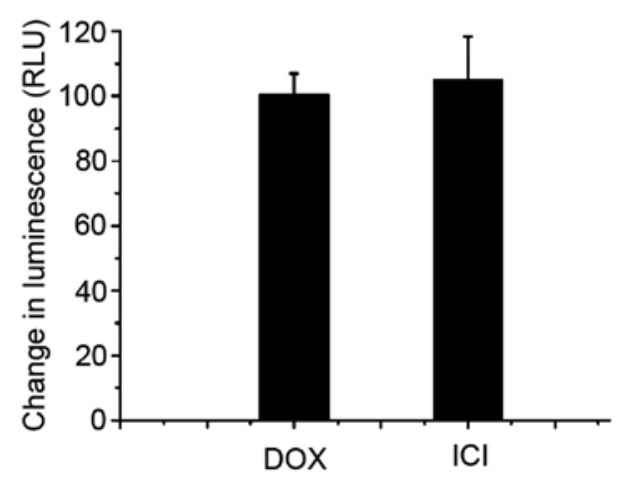

Figure 4. Function of fulvestrant as a substrate for transport by P-gp. $5 \mu \mathrm{M}$ fulvestrant or $5 \mu \mathrm{M}$ doxorubicin-treated P-gp reactions were performed according to the manufacturer's protocol. Luminescence was read on a luminometer. The decrease in the average relative light units of samples ( $\triangle R L U)$ represents fulvestrant or doxorubicin-stimulated P-gp ATPase activity. CTL, control; ICI, fulvestrant; DOX, doxorubicin.
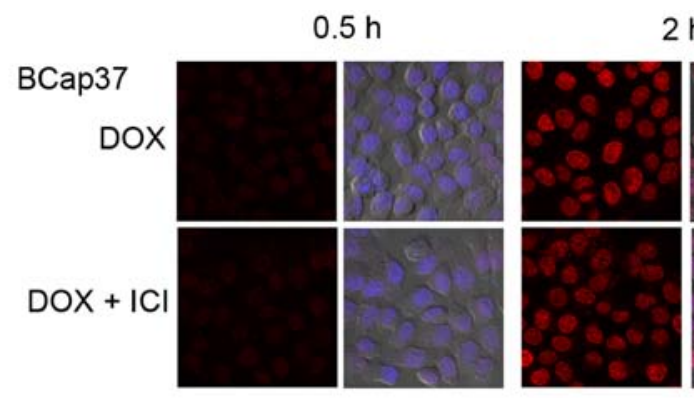

$2 \mathrm{~h}$
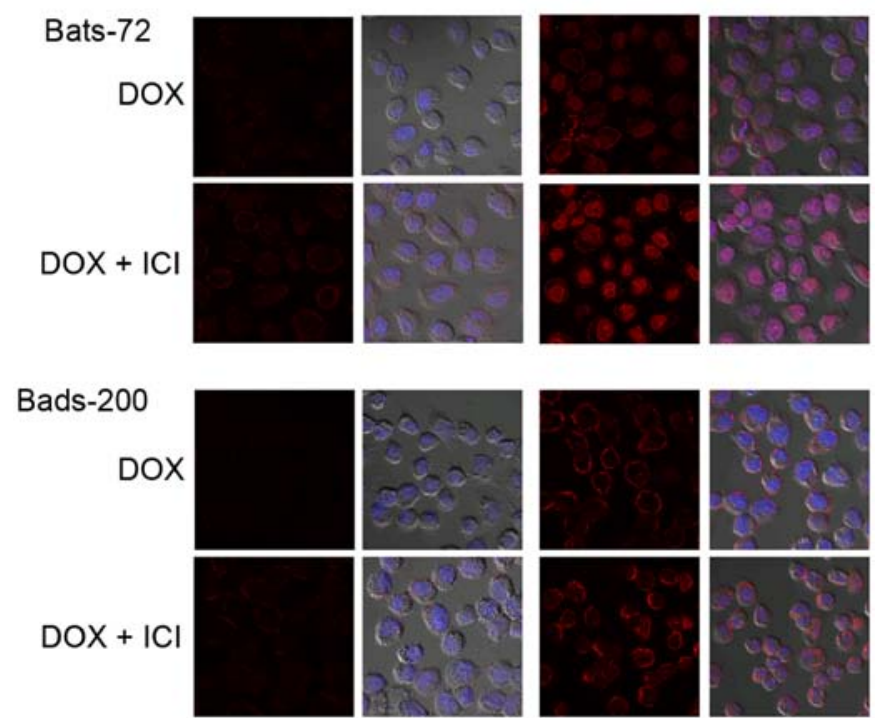

Figure 5. Fulvestrant alters intracellular doxorubicin distribution and accumulation. In vitro confocal images of doxorubicin fluorescence distribution in the nuclei and cytoplasm. Cells pretreated or non-pretreated with $5 \mu \mathrm{M}$ fulvestrant were incubated with $5 \mu \mathrm{M}$ doxorubicin for 0.5 and $2 \mathrm{~h}$ respectively. After cell nuclei was labeled with Hoechst-33342, fluorescent signals for doxorubicin (red) and Hoechst-33342 (blue) were observed by confocal microscopy at x 600 magnification. ICI, fulvestrant; DOX, doxorubicin.

the interaction between fulvestrant and P-gp which acts as an ATP-dependent efflux pump and relies on ATP hydrolysis, the effect of fulvestrant on P-gp ATPase activity was measured next. As Fig. 4 shows, fulvestrant significantly stimulated the P-gp ATPase activity like doxorubicin which means
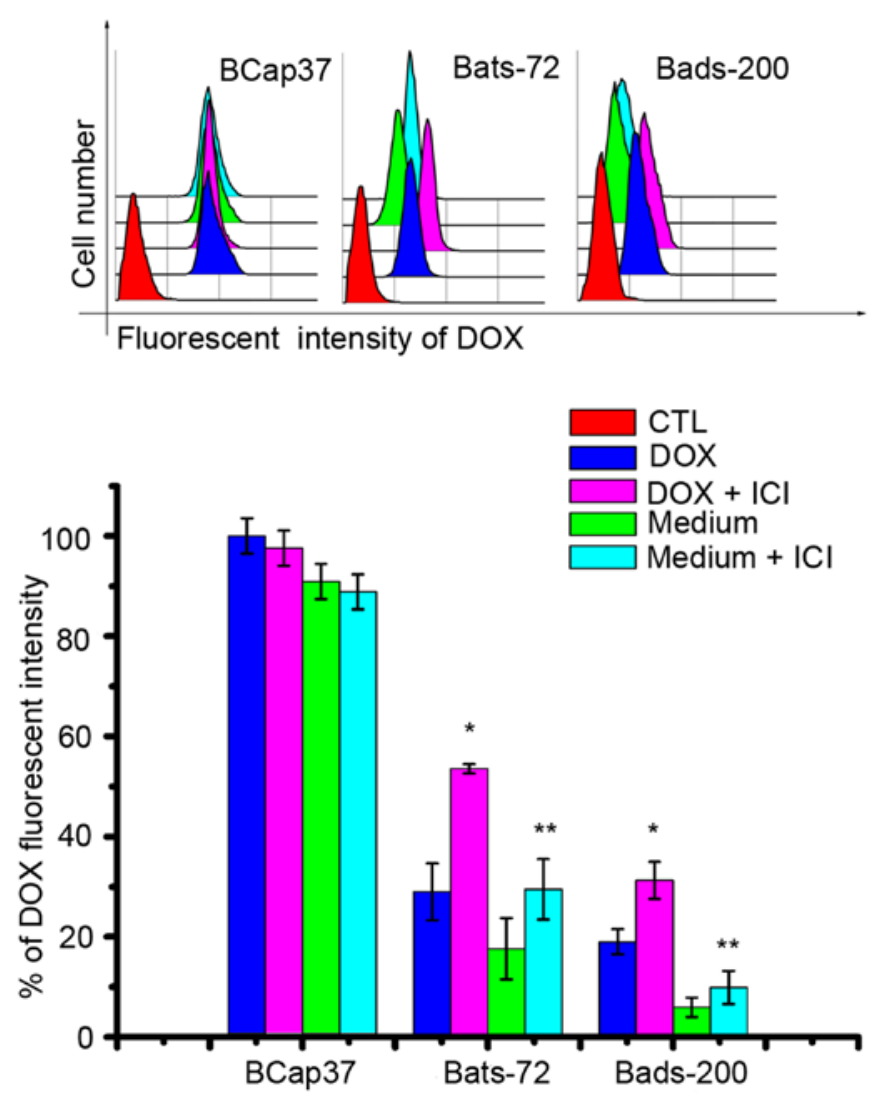

Figure 6. Fulvestrant alters intracellular doxorubicin uptake and efflux. Quantitation of doxorubicin uptake and efflux by flow cytometric analysis. Cells pretreated or non-pretreated with $5 \mu \mathrm{M}$ fulvestrant were incubated with $5 \mu \mathrm{M}$ doxorubicin for $2 \mathrm{~h}$ (uptake); After treated with the combination of doxorubicin and fulvestrant for $2 \mathrm{~h}$, cells were washed three times with icecold PBS and then incubated with drug-free medium or medium with $5 \mu \mathrm{M}$ fulvestrant for another $2 \mathrm{~h}$ (efflux). The intracellular fluorescence intensity of doxorubicin was measured by flow cytometry. The ratio of mean fluorescent intensity in BCap37 cells exposed to DOX for $2 \mathrm{~h}$ was $100 \%$; ${ }^{*} \mathrm{P}<0.05$ vs. DOX, ${ }^{* *} \mathrm{P}<0.05$ vs. medium. CTL, control, represents cells incubated without DOX; ICI, fulvestrant; DOX, doxorubicin.

fulvestrant is a substrate for transport by P-gp. Interestingly, the decrease in luminescence (the average relative light units $\Delta \mathrm{RLU})$ of $5 \mu \mathrm{M}$ fulvestrant-treated samples was approximately $105.17 \pm 13.21$, which is almost equal to that of $5 \mu \mathrm{M}$ doxorubicin-treated samples which was $100.73 \pm 6.18$. These data demonstrate fulvestrant reverses doxorubcin resistance as a substrate of P-gp that inhibits its role of drug-efflux pump.

Fulvestrant alters intracellular doxorubicin distribution, accumulation and retention. Using confocal fluorescent microscopy, we observed the doxorubicin auto-fluorescent intensity to assess the intracellular doxorubicin distribution and accumulation in BCap37, Bats-72 and Bads-200 cells. Fig. 5 indicated that intracellular doxorubicin which increased in a time-dependent manner were mostly accumulated in nuclei of the parental BCap37 cells, but localized both in the nuclei and cytoplasm of Bats-72 and Bads-200 cells. Co-treatment of fulvestrant increased doxorubicin accumulation and relocalized it to the nuclei in Bats-72 and Bads-200 cells. Quantitation of doxorubicin uptake and efflux was measured by flow cytometric analysis. As Fig. 6 illustrates, the doxorubicin uptake was slower but efflux was faster in Bats-72 
and Bads-200 cells than in BCap37 cells. Incubation with the addition of fulvestrant increased doxorubicin uptake in Bats-72 $(28.97 \pm 5.68$ versus $53.52 \pm 0.94 \%, \mathrm{P}<0.05)$ and Bads-200 cells $(18.99 \pm 2.49$ versus $31.27 \pm 3.68 \%, \mathrm{P}<0.05)$, and incubation with doxorubicin-free medium for another $2 \mathrm{~h}$ after that co-treatment, fulvestrant also can inhibited efflux and increased

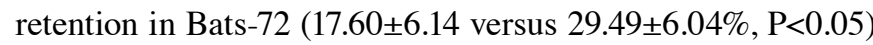
and Bads-200 cells $(5.94 \pm 1.93$ versus $9.86 \pm 3.3 \%, \mathrm{P}<0.05)$. These findings suggested fulvestrant increased intracellular doxorubicin accumulation and retention and relocalized it to the nuclei in Bats-72 and Bads-200 cells, but had no significant influence in parental BCap37 cells.

\section{Discussion}

By a series of cytotoxicity assays in vitro, we found that fulvestrant significantly sensitized doxorubicin-induced cytotoxicity in a dose-dependent manner in ER-negative MDR cell lines including Bats-72, Bads-200 cell lines and KBv200 cells. Direct comparison with known modulators further elucidated that the reversal potency of fulvestrant is similar to that of verapamil and more potent than that of tamoxifen when administered at the same doses in vitro. In addition, compared to other MDR modulators, another prominent feature of fulvestrant is its safety. The concentration of fulvestrant required to achieve a marked reversal has little cytotoxicity by itself. Clinical observations further confirmed this conclusion, fulvestrant which is a pure ER antagonist possesses no agonist effects, while tamoxifen is thought to be a partial estrogen agonist. Estrogen side effects may cause endometrial hyperplasia or cancer, uterine sarcoma, and may increase the risk of deep vein thrombosis and stroke (30-32). Considering the effective and well-tolerated properties, fulvestrant is a promising modulator for the treatment for $\mathrm{P}$-gp-mediated drug resistance.

Cumulative evidence suggests that resistance to cell death programs and cell cycle arrest also contributes to the development of MDR (33-35). Our data indicate that the extent of doxorubicin-induced apoptosis and G2/M arrest is closely related to the potency of drug resistance in BCap37, Bats-72, Bads-200 cell lines. Fulvestrant significantly potentiated doxorubicin-induced apoptosis and G2/M arrest in Bats-72 and Bads-200 cell lines, while it alone did not induce apoptosis or change cell cycle progression, probably representing fulvestrant restored sensitivity to doxorubicin in Bats-72 and Bads-200 cell lines partially through regulation of cell death and cell cycle pathways. The western blot analyses further indicated that the doxorubicin-induced $\mathrm{G} 2 / \mathrm{M}$ arrest was more likely mediated by the level of cyclin B1, and fulvestrant enhanced doxorubicin-induced G2/M arrest through upregulation of cyclin B1 expression. It is well known that alterations of the levels of Bcl-2 family proteins play an active role in apoptotic pathways $(36,37)$. It seemed that the levels of the major anti-apoptotic protein $\mathrm{Bcl}-2$ which remained stable with or without doxorubicin did not associate with the sensitivity to doxorubicin-induced apoptosis.

The interaction between fulvestrant and P-gp was further investigated. The ATPase assay showed fulvestrant could stimulate the ATPase activity of P-gp, which means that it acts as a substrate of P-gp to inhibit its function of drug-efflux possibly by competitively binding to P-gp. Western blotting further indicated that fulvestrant did not alter P-gp expression. Therefore, fulvestrant could modulate P-gp mediated resistance mainly by inhibiting its function, and not by inhibiting its expression. On the other hand, fulvestrant increased intracellular doxorubicin accumulation and retention in Bats-72 and Bads-200 cells, but had no significant influence in parental BCap37 cells that lack P-gp, also implied that the reversal of drug resistance by fulvestrant was probably attributable to the inhibition of P-gp-mediated drug transport. Interestingly, we observed that Bats-72 and Bads-200 cells altered intracellular doxorubicin distribution and accumulation compared with parental BCap37 cells. Confocal cell images displayed that intracellular doxorubicin was mostly concentrated in nuclei of the parental BCap37 cells, but localized both in the nuclei and cytoplasm of Bats-72 and Bads-200 cells, especially for Bads-200 cells, the majority of doxorubicin was still in the cytoplasm. Those results suggested that doxorubicin could not easily get access to nuclear targets of MDR cells. Fulvestrant not only restored doxorubicin accumulation but also tried to relocalize it to the nuclei in Bats- 72 and Bads-200 cells. The mechanism of doxorubicin activity is thought to interact with DNA by intercalation (38-40), so fulvestrant increased the amount of doxorubicin accessed to nuclear targets in MDR cells, which maybe another potential reason related to its reversal potency to $\mathrm{P}$-gp mediated doxorubicin resistance.

In conclusion, we have shown that fulvestrant significantly reverses P-gp mediated resistance to doxorubcin in vitro. The results suggest that fulvestrant not only takes part in ER-mediated pathway for breast cancer therapy, but also has an important role in the reversal of drug resistance when combined with chemotherapy agents independent of ER expressing. This study may provide useful clues for understanding the novel anticancer mechanism of fulvestrant and supporting the clinical application of fulvestrant when added to chemotherapy regimens for the treatment of metastatic and progressive breast cancer, however, further research is still needed to determine the ideal combination therapy strategy.

\section{Acknowledgements}

This study was supported by grants LQ14H160004 from Zhejiang Provincial Natural Science Foundation of China and NSFC-81302288 from National Natural Science Foundation of China.

\section{References}

1. Jemal A, Bray F, Center MM, Ferlay J, Ward E and Forman D: Global cancer statistics. CA Cancer J Clin 61: 69-90, 2011.

2. McGuire WL, Horwitz KB, Pearson OH and Segaloff A: Current status of estrogen and progesterone receptors in breast cancer. Cancer 39 (Suppl): S2934-S2947, 1977.

3. Dickson RB and Lippman ME: Estrogenic regulation of growth and polypeptide growth factor secretion in human breast carcinoma. Endocr Rev 8: 29-43, 1987.

4. Kuukasjärvi T, Kononen J, Helin H, Holli K and Isola J: Loss of estrogen receptor in recurrent breast cancer is associated with poor response to endocrine therapy. J Clin Oncol 14: 2584-2589, 1996.

5. Lück HJ and Roché H: Weekly paclitaxel: An effective and welltolerated treatment in patients with advanced breast cancer. Crit Rev Oncol Hematol 44 (Suppl): S15-S30, 2002. 
6. Sledge GW, Neuberg D, Bernardo P, Ingle JN, Martino S, Rowinsky EK and Wood WC: Phase III trial of doxorubicin, paclitaxel, and the combination of doxorubicin and paclitaxel as front-line chemotherapy for metastatic breast cancer: An intergroup trial (E1193). J Clin Oncol 21: 588-592, 2003.

7. Andre F and Pusztai L: Molecular classification of breast cancer: Implications for selection of adjuvant chemotherapy. Nat Clin Pract Oncol 3: 621-632, 2006.

8. Dean M, Fojo T and Bates S: Tumour stem cells and drug resistance. Nat Rev Cancer 5: 275-284, 2005.

9. Buzdar A, Jonat W, Howell A, Jones SE, Blomqvist C, Vogel CL, Eiermann W, Wolter JM, Azab M, Webster A, et al; Arimidex Study Group: Anastrozole, a potent and selective aromatase inhibitor, versus megestrol acetate in postmenopausal women with advanced breast cancer: Results of overview analysis of two phase III trials. J Clin Oncol 14: 2000-2011, 1996.

10. Dombernowsky P, Smith I, Falkson G, Leonard R, Panasci L, Bellmunt J, Bezwoda W, Gardin G, Gudgeon A, Morgan M, et al: Letrozole, a new oral aromatase inhibitor for advanced breast cancer: Double-blind randomized trial showing a dose effect and improved efficacy and tolerability compared with megestrol acetate. J Clin Oncol 16: 453-461, 1998.

11. Lønning PE, Bajetta E, Murray R, Tubiana-Hulin M,Eisenberg PD, Mickiewicz E, Celio L, Pitt P, Mita M, Aaronson NK, et al Activity of exemestane in metastatic breast cancer after failure of nonsteroidal aromatase inhibitors: A phase II trial. J Clin Oncol 18: 2234-2244, 2000.

12. Vergote I and Robertson JF: Fulvestrant is an effective and welltolerated endocrine therapy for postmenopausal women with advanced breast cancer: Results from clinical trials. Br J Cancer 90 (Suppl 1): S11-S14, 2004.

13. Dowsett M, Nicholson RI and Pietras RJ: Biological characteristics of the pure antiestrogen fulvestrant: Overcoming endocrine resistance. Breast Cancer Res Treat 93 (Suppl 1): S11-S18, 2005.

14. Osborne CK, Wakeling A and Nicholson RI: Fulvestrant: An oestrogen receptor antagonist with a novel mechanism of action. Br J Cancer 90 (Suppl 1): S2-S6, 2004.

15. Howell A: The future of fulvestrant ('Faslodex'). Cancer Treat Rev 31 (Suppl 2): S26-S33, 2005.

16. Shen H, Liu J, Wang R, Qian X, Xu R, Xu T, Li Q, Wang L, Shi Z, Zheng J, et al: Fulvestrant increases gefitinib sensitivity in non-small cell lung cancer cells by upregulating let-7c expression. Biomed Pharmacother 68: 307-313, 2014.

17. Sui M, Huang Y, Park BH, Davidson NE and Fan W: Estrogen receptor alpha mediates breast cancer cell resistance to paclitaxel through inhibition of apoptotic cell death. Cancer Res 67: 5337-5344, 2007.

18. Sui M, Jiang D, Hinsch C and Fan W: Fulvestrant (ICI 182,780) sensitizes breast cancer cells expressing estrogen receptor alpha to vinblastine and vinorelbine. Breast Cancer Res Treat 121 335-345, 2010.

19. Chang J, Sui M and Fan W: Estrogen receptor $\alpha$ attenuates therapeutic efficacy of paclitaxel on breast xenograft tumors. Breast Cancer Res Treat 134: 969-980, 2012.

20. Jiang D, Sui M, Zhong W, Huang Y and Fan W: Different administration strategies with paclitaxel induce distinct phenotypes of multidrug resistance in breast cancer cells. Cancer Lett 335 : 404-411, 2013.

21. Roepe PD: The P-glycoprotein efflux pump: How does it transport drugs? J Membr Biol 166: 71-73, 1998.

22. Sauna ZE, Kim IW and Ambudkar SV: Genomics and the mechanism of P-glycoprotein (ABCB1). J Bioenerg Biomembr 39: 481-487, 2007.

23. Ambudkar SV, Kimchi-Sarfaty C, Sauna ZE and Gottesman MM: P-glycoprotein: from genomics tomechanism. Oncogene 22 7468-7485, 2003.
24. Jiang D, Huang Y, Han N, Xu M, Xu L, Zhou L, Wang S and Fan W: Fulvestrant, a selective estrogen receptor down-regulator, sensitizes estrogen receptor negative breast tumors to chemotherapy. Cancer Lett 346: 292-299, 2014.

25. Zhu X, Sui M and Fan W: In vitro and in vivo characterizations of tetrandrine on the reversal of P-glycoprotein-mediated drug resistance to paclitaxel. Anticancer Res 25B: 1953-1962, 2005.

26. Duan Z, Choy E and Hornicek FJ: NSC23925, identified in a high-throughput cell-based screen, reverses multidrug resistance. PLoS One 4: e7415, 2009.

27. Ren D, Zhu Q, Li J, Ha T, Wang X and Li Y: Overexpression of angiopoietin-1 reduces doxorubicin-induced apoptosis in cardiomyocytes. J Biomed Res 26: 432-438, 2012.

28. Rebbaa A, Zheng X, Chou PM, and Mirkin BL: Caspase inhibition switches doxorubicin-induced apoptosis to senescence Oncogene 22: 2805-2811, 2003.

29. Zuryñ A, Litwiniec A, Gackowska L, Pawlik A, Grzanka AA and Grzanka A: Expression of cyclin A, B1 and D1 after induction of cell cycle arrest in the Jurkat cell line exposed to doxorubicin. Cell Biol Int 36: 1129-1135, 2012.

30. Hu R, Hilakivi-Clarke L and Clarke R: Molecular mechanisms of tamoxifen-associated endometrial cancer (Review). Oncol Lett 9: 1495-1501, 2015

31. Min CR, Kim MJ, Park YJ, Kim HR, Lee SY, Chung KH and Oh SM: Estrogenic effects and their action mechanism of the major active components of party pill drugs. Toxicol Lett 214: 339-347, 2012.

32. Iqbal J, Ginsburg OM, Wijeratne TD, Howell A, Evans G, Sestak I and Narod SA: Endometrial cancer and venous thromboembolism in women under age 50 who take tamoxifen for prevention of breast cancer: A systematic review. Cancer Treat Rev 38: 318-328, 2012.

33. Feng $\mathrm{R}$ and Dong L: Knockdown of microRNA-127 reverses adriamycin resistance via cell cycle arrest and apoptosis sensitization in adriamycin-resistant human glioma cells. Int J Clin Exp Pathol 8: 6107-6116, 2015.

34. Efferth T, Fabry U and Osieka R: Apoptosis and resistance to daunorubicin in human leukemic cells. Leukemia 11: 1180-1186, 1997.

35. De U, Chun P, Choi WS, Lee BM, Kim ND, Moon HR, Jung JH and Kim HS: A novel anthracene derivative, MHY412, induces apoptosis in doxorubicin-resistant MCF-7/Adr human breast cancer cells through cell cycle arrest and downregulation of P-glycoprotein expression. Int J Oncol 44: 167-176, 2014.

36. Kelly PN and Strasser A: The role of Bcl-2 and its pro-survival relatives in tumourigenesis and cancer therapy. Cell Death Differ 18: 1414-1424, 2011.

37. Llambi F and Green DR: Apoptosis and oncogenesis: Give and take in the BCL-2 family. Curr Opin Genet Dev 21: 12-20, 2011.

38. Bodley A, Liu LF, Israel M, Seshadri R, Koseki Y, Giuliani FC, Kirschenbaum S, Silber R and Potmesil M: DNA topoisomerase II-mediated interaction of doxorubicin and daunorubicin congeners with DNA. Cancer Res 49: 5969-5978, 1989.

39. Cirilli M,BachechiF,Ughetto G,ColonnaFP and Capobianco ML: Interactions between morpholinyl anthracyclines and DNA. The crystal structure of a morpholino doxorubicin bound to d(CGTACG). J Mol Biol 230: 878-889, 1993.

40. Kellogg GE, Scarsdale JN and Fornari FA Jr: Identification and hydropathic characterization of structural features affecting sequence specificity for doxorubicin intercalation into DNA double-stranded polynucleotides. Nucleic Acids Res 26: 4721-4732, 1998 